\title{
Thermal Diapirism and the Habitability of the Icy Shell of Europa
}

\author{
Javier Ruiz • Lilia Montoya • Valle López • Ricardo Amils
}

Received: 11 January 2007 / Accepted: 19 February 2007 /

Published online: 15 March 2007

\begin{abstract}
Europa's chaos and lenticulae features may have originated by thermal diapirs related to convective plumes. Warm ice plumes could be habitable, since their temperature is close to the ice melting temperature. Moreover, thermal plumes intruding into the lower stagnant lid warm several kilometers of country ice above $230 \mathrm{~K}$ for periods of $10^{5}$ years, and hundreds of meters above $240 \mathrm{~K}$ for periods of $10^{4}$ years. Diapir coalescence generating chaos areas should provide a large zone with temperature above $\sim 240 \mathrm{~K}$ for thousands of years. A temperature above $\sim 230 \mathrm{~K}$ is potentially interesting for astrobiology, since it corresponds to the lowest temperature at which microbial metabolic activity in Antarctic ice has been reported. So, the warming by thermal plumes could cause an aureole of biological activation/reactivation in the country ice. Adaptation of life to either high salinity or low temperature is similar: it requires the synthesis of compatible solutes, like trehalose or glycerol, which are efficient cryoprotectants. We therefore propose that the future astrobiological exploration of Europa should include the search for compatible solutes in chaos and lenticulae features.
\end{abstract}

Keywords Astrobiology · Compatible solutes · Europa · Habitability · Thermal diapirism

\footnotetext{
J. Ruiz $(\bowtie)$

Instituto de Astrofísica de Andalucía, CSIC, Camino Bajo de Huétor 50, 18008 Granada, Spain e-mail: ruiz@iaa.es

L. Montoya $\cdot$ R. Amils

Centro de Biología Molecular, CSIC-Universidad Autónoma de Madrid, 28049 Cantoblanco, Madrid, Spain

V. López

Seminario de Ciencias Planetarias, Facultad de Ciencias Geológicas, Universidad Complutense de Madrid, 28040 Madrid, Spain

R. Amils

Centro de Astrobiología, INTA, 28855 Torrejón de Ardoz, Madrid, Spain
} 


\section{Introduction}

Biological organisms may exist, active or latent, inside the icy shell of Europa, at places such as fractures, veins or liquid reservoirs (e.g., Greenberg et al. 2000; Marion et al. 2003; Lipps and Rieboldt 2005). Beneath this shell an internal ocean may exist, as suggested by geological evidence (e.g., Pappalardo et al. 1999), and strongly implied by the magnetic signature of a conductive electric layer within a few tens of kilometers at most of the surface (Kivelson et al. 2000; Schilling et al. 2004). This internal ocean could be habitable (e.g., Reynolds et al. 1983; Chyba 2000; Chyba and Phillips 2001, 2002; Schulze-Makuch and Irwin 2002; Marion et al. 2003). The shell composition, and hence the potential habitats, may include, at least locally, large amounts of non-water ice components, maybe magnesium and sodium sulfate hydrates (e.g., McCord et al. 1998; Kargel et al. 2000), sulfuric acid (Carlson et al. 1999), or mixtures of both (Spencer et al. 2006).

The icy shell is an evolving environment that should affect the viability, activity and evolution of any possible organism or biological community living inside. Geological mapping suggests that surface deformation and modification styles have changed through the recorded geological history from tectonic resurfacing, with ridge plains and bands formation, to terrain disruption that would lead to chaos areas and lenticulae, including domes, pits, and microchaos features (e.g., Prockter et al. 1999; Kadel et al. 2000; Figueredo and Greeley 2004; for a review of the Europa geology see Greeley et al. 2004) (Fig. 1).

This change in geological characteristics seems to be related to a progressive thickening of the icy shell. For example, younger ridges are wider, higher and further apart than older ones (Kadel et al. 2000; Figueredo and Greeley 2004). The most popular explanation for the origin of domes and microchaos features is surface upwarping and disruption by thermal diapirism related to convection in the lower part of the icy shell (Pappalardo et al. 1998; Pappalardo and Head 2001). In addition, differential tidal heating of warm ice or compositional differences could contribute to the buoyancy of upwelling plumes (Sotin et al. 2002; Pappalardo and Barr 2004). In this scenario, large chaos areas would be formed by coalescence of diapirs (Spaun et al. 1998; Schenk and Pappalardo 2004). Convection in the icy shell starts when the shell is sufficiently thick (e.g., McKinnon 1999), and then a

Fig. 1 Galileo image showing the chaotic area named Conamara Chaos (upper right corner) and numerous features collectively referred to as lenticulae, which include microchaos and domes. The area dimensions are $100 \times 140 \mathrm{~km}$

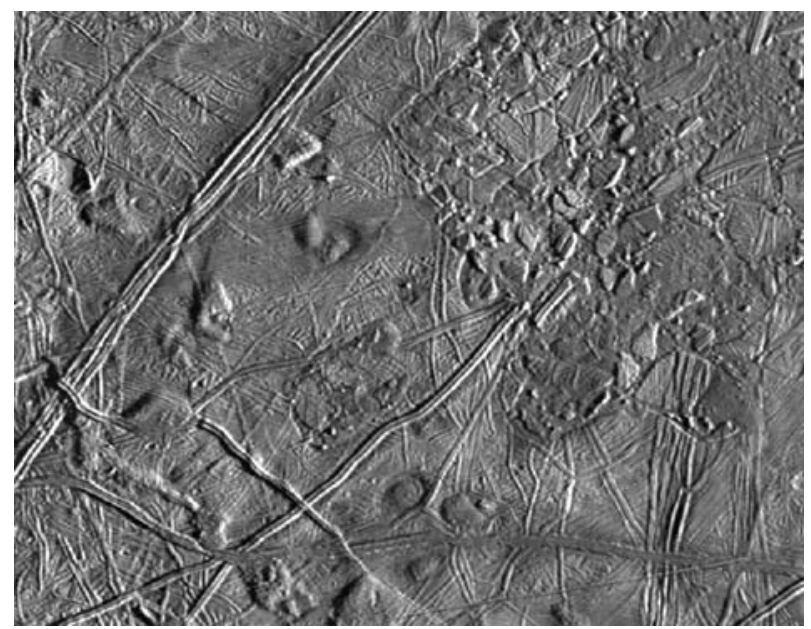


stagnant, cold and thermally conductive lid develops above the actively convective layer. Thermal diapirism would imply putting into contact the cold stagnant lid with ice plumes whose temperatures are close to the ice melting temperature (convective upwellings originate in the lower boundary layer of the convective system). This would have significant implications for the habitability of the upper part of the ice shell of Europa, as well as for the evolution of putative europan ecosystems. Thus, in this paper we consider the effect of thermal diapirism on the habitability of the ice shell of Europa, as well as its possible implications for astrobiological exploration.

\section{The Lowest Temperature for Biological Activity and the Habitability of the Stagnant Lid}

Price and Sowers (2004) have recently discussed evidence for microbial metabolic activity in Antarctic glacial ice at $-40^{\circ} \mathrm{C}$. Those authors found no threshold temperature below which metabolism would cease, and they found similar metabolic rates (for equal temperature) for microbes in water, soil, sediment, permafrost or ice. These results notably lower the minimum temperature for microbial metabolism below the usually cited value of around $-20^{\circ} \mathrm{C}$ (e.g., Rothschild and Mancinelli 2001; Marion et al. 2003). As a consequence, life on Europa is more likely than before.

Thus, here we consider ice above $\sim 230 \mathrm{~K}$ as potentially interesting for astrobiology. A small amount of liquid water can be shown to be available in veins in Europa's ice shell at temperatures as low as $230 \mathrm{~K}$, since the presence of non-water ice substances lowers the melting point of the ice. For example, the eutectic of the water-sulfuric acid is $211 \mathrm{~K}$ (e.g., Kargel et al. 2000), and chloride salts can have a eutectic as low as 223-230 K (e.g., Bodnar 2001). Similarly, liquid water at similar temperatures exists in terrestrial polar ice and permafrost (e.g., Dash et al. 1995; Price 2000). Deming (2002) has speculated that life could be possible as long as liquid water is available, and she has coined the term "eutectophiles" for organisms living at liquid veins or reservoirs close to the eutectic point of water containing impurities. Although it is an interesting possibility, here we limit our considerations to temperatures above $\sim 230 \mathrm{~K}$.

The temperature at the base of the stagnant lid would be between 230 and $250 \mathrm{~K}$, depending on the convective model and adopted ice rheology (e.g., Ruiz and Tejero 2003; Tobie et al. 2003). Due to these low temperatures, the possible biological activity inside the stagnant lid would be restricted, at most, to its lowest part, although convection in the ice shell could facilitate communication and material interchange between the base of the stagnant lid and the internal ocean. Thermal upwelling plumes transport materials (which could include nutrients, metabolic products, or microorganisms) from the lowest ice shell, and potentially from the ice shell/internal ocean interphase, to the upper ice shell, and even to the surface if the terrain is sufficiently disrupted (Pappalardo and Head 2001; Lipps and Rieboldt 2005). Thermal diapirs might themselves be habitable (Price 2003). The initial temperature of a thermal upwelling in the ice shell would be at least $\sim 260 \mathrm{~K}$, a typical value for the nearly isothermal interior of the convective layer (McKinnon 1999). This temperature permits microbial life in terrestrial ice environments (see Marion et al. 2003, and references therein). Subsequent plume cooling reduces (or even suppresses) the potential for life.

Thermal plumes must also have thermal effects in the country ice where they intrude, and they could generate transient brines (Pappalardo et al. 1998). The total lifetime for thermal diapirs with a radius of a few kilometers is $\sim 10^{7}$ years at most (Gaidos et al. 1999; 
see also the next section). For this reason, Gaidos et al. (1999) have considered it unlikely that a global biosphere could be sustained in this way, although in any case thermal diapirs could have effects on habitability in the vicinity of plumes. The thermal history of an ice plume and its surroundings is analyzed in the next section.

\section{Warming by Thermal Plumes}

Figure 2 shows the thermal history of a vertical cylindrical intrusion of square section and its effects on the country ice. Calculations were performed using the classical theory for thermal histories of intrusions and their surroundings (e.g., Jaeger 1964, 1968). We used a vertical intrusion since it is a good analog for a thermal plume. Results for cylinders with square cross-section are very similar to those obtained for circular cylinders (e.g., Jaeger 1968), and the mathematical treatment is simpler. This simplification is justified since our goal is to obtain an approximate estimation of the warming effect instead of a detailed modeling.

For a vertical intrusion of square cross-section, and assuming equal thermal properties for the country and intrusive ice, the temperature at the time $t$ following the intrusion

Fig. 2 Thermal history of an ice plume and their surroundings, shown for two ambient temperatures. The ice plume is assumed to be cylindrical, with square section, and $6 \mathrm{~km}$ width. Distances are given perpendicular to the face midpoints. Labels indicate time in years, and the intrusive process is instantaneous
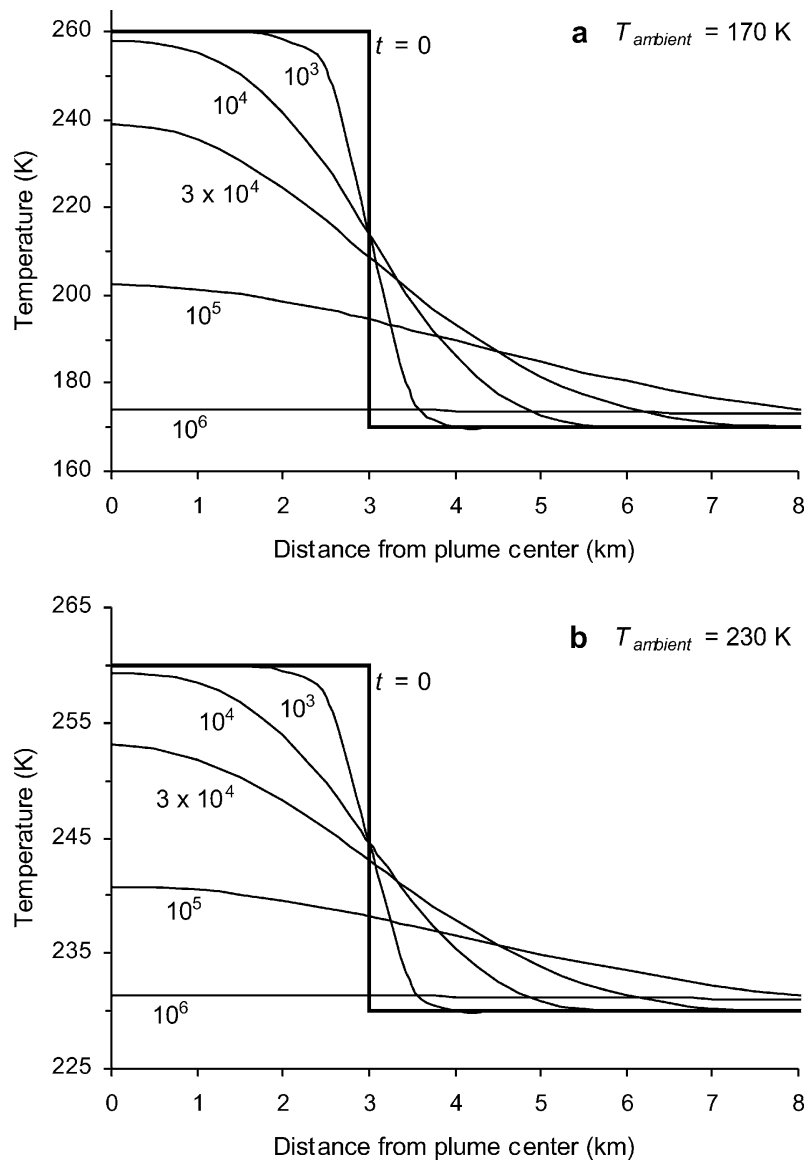
(which is considered to occur instantaneously at $t=0$ ), at a point with coordinates $x, y$, is given by

$$
T(x, y, t)=T_{0}+\left(T_{i}-T_{0}\right) \phi(x, t) \varphi(y, t),
$$

where $x$ and $y$ are the distances along the corresponding axes, which are perpendicular to the face midpoints (the origin of coordinates is in the center of the figure section), $T_{\mathrm{o}}$ is the temperature of the initial country material, and $T_{i}$ is the initial temperature of the intrusion. The function $\phi(x, t)$ is given by

$$
\phi(x, t)=\frac{1}{2}\left[\operatorname{erf}\left(\frac{x+a}{2(\kappa t)^{1 / 2}}\right)-\operatorname{erf}\left(\frac{x-a}{2(\kappa t)^{1 / 2}}\right)\right],
$$

where

$$
\operatorname{erf}(u)=\frac{2}{\sqrt{\pi}} \int_{0}^{u} e^{-w^{2}} \mathrm{dw}
$$

$a$ is the plume half-width and $\kappa$ is the thermal diffusivity; the function $\varphi(y, t)$, for $y=0$ (temperatures calculated along axis $x$ ), is

$$
\varphi(y, t)=\frac{1}{2}\left[\operatorname{erf}\left(\frac{a}{2(\kappa t)^{1 / 2}}\right)-\operatorname{erf}\left(\frac{-a}{2(\kappa t)^{1 / 2}}\right)\right] .
$$

These calculations do not include thermal effects due to the upper and lower ends of the intrusion, since these effects have limited influence on lateral temperature variations.

The calculations have been performed for a square plume $6 \mathrm{~km}$ width (the size of the lenticulae is typically between 4 and $8 \mathrm{~km}$; Spaun et al. 2004), with an initial temperature of $260 \mathrm{~K}$ (typical of the actively convective layer; McKinnon 1999), intruding into country ice with temperature of 170 or $230 \mathrm{~K}$. The higher value is representative of both the lower part of the stagnant lid and the lower temperature in which metabolic activity has been reported; in turn, $170 \mathrm{~K}$ is the approximate intermediate value between the surface temperature $(\sim 100 \mathrm{~K})$ and the temperature at the base of the stagnant lid. Finally, the coefficient of thermal diffusion was taken as $2 \times 10^{-6} \mathrm{~m}^{2} \mathrm{~s}^{-2}$, a value roughly valid for water ice near to $200 \mathrm{~K}$ (see for example Kirk and Stevenson 1987).

Figure 2 shows that warming of the country ice by thermal plumes is limited. However, convective plumes intruding close to the stagnant lid base can warm several kilometers of country ice above $230 \mathrm{~K}$ during periods of $10^{5}$ years, and hundreds of meters above $240 \mathrm{~K}$ during periods of $10^{4}$ years. Consequently, if there are organisms inside the ice shell, a thermal plume could cause an aureole of biological activation or reactivation in the country ice, in a way similar to that by which a terrestrial magmatic intrusion causes an aureole of contact metamorphism.

If chaos areas have originated due to coalescence of diapirs, then the disrupted ice in these areas may be an adequate habitat for microorganisms. Indeed, close packing of intrusions produces a generalized rise of temperature, which peaks close to the mean temperature between the initial country material and intrusion temperatures (see Jaeger 1968). Diapirs that have coalesced near the base of the stagnant lid should therefore give an extended zone (even hundreds of kilometers across) above $\sim 240 \mathrm{~K}$ during tens of thousands of years. 


\section{Discussion and Implications for Astrobiological Exploration}

It has been suggested that variations in orbital parameters could cause episodic changes in tidal heating and hence in ice shell dynamics and thickness, with oscillations between a thin and thermally conductive state and a thick and convective one (Hussmann and Spohn 2004). If the ice shell is thick and convective, an increase of eccentricity could generate so much tidally dissipated heat that it could not be efficiently transported by convection. In this case, the ice shell heats, melts from this base and thins, and convection ceases. As a consequence, thermal diapirism, and material interchange between the stagnant lid and the internal ocean potentially driven by convection ceases too (although liquid water could ascent through fractures). A subsequent decrease of eccentricity would lead to a progressive cooling and thickening of the ice shell, and to convection and thermal diapirism and their potential effects for biological activity. Thus, the habitability conditions could cyclically vary in the ice shell of Europa.

Chaos areas are considered to be favorable places for the search of biomarkers on Europa, based on evidence for material interchange between surface and subsurface, presence of non-ice components, and possibly melting (e.g., Chyba and Phillips 2001; Figueredo et al. 2003). A similar argument can be proposed for individual lenticulae and microchaos features. Additionally, terrain disruption leading to chaos and microchaos features would favor the arrival of organisms and/or metabolic products at the surface, especially if the stagnant lid is only a few kilometers thick, as suggested by high surface heat flow deduced from geological indicators (Ruiz and Tejero 2000; Ruiz 2005), or deformation induced by plume upwellings (Nimmo and Manga 2002).

Life at very low temperature requires the presence of cryoprotectant substances. It is known that terrestrial life at low temperature is adapted to low water activity in the same way as it is adapted to high osmolarity or desiccation conditions (e.g., Potts 1994; Thomas and Dieckmann 2002). This implies that a decrease in temperature would be equivalent to an increase in osmolarity or desiccation. A typical physiological response to these conditions

Fig. 3 Intracellular accumulation of compatible solutes by organisms living in hypothetical habitats within Europa's ice shell: a the stagnant lid warmed by thermal diapirs, and $\mathbf{b}$ the diapirs themselves. Dots indicate the relative importance of both compatible solute accumulation and their interchange with the environments

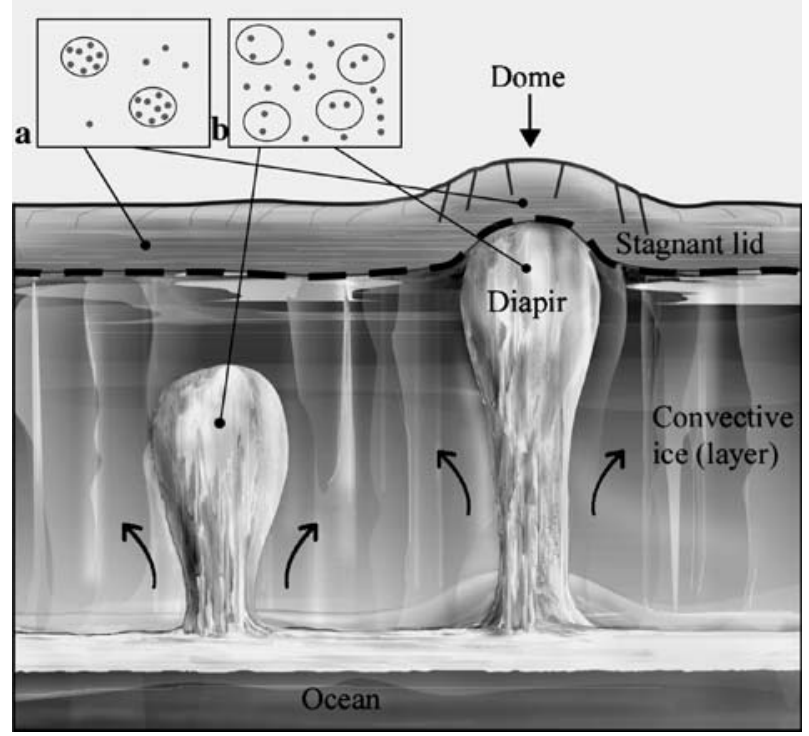


is intracellular accumulation of compatible solutes, which can reach high intracellular concentrations (Kempf and Bremer 1998; DasSarma and Arora 2002; Elbein et al. 2003). In consequence, the synthesis of compatible solutes, like trehalose, glycerol or glycine betaine, in response to freezing conditions could result in an important survival strategy for any organism living within the ice shell of Europa (Fig. 3), since these substances are efficient cryoprotectants (Welsh 2000; Elbein et al. 2003; Cleland et al. 2004; Yancey 2005). In a similar way, Stan-Lotter et al. (2003) have experimentally shown that the presence of glycerol enhances the survival rate of halophilic archeobacteria during deep freezingdrying in simulated Martian atmosphere conditions.

The synthesis of compatible solutes is widely observed on the Earth, and it has originated several times in distant phylogenetic groups, for example methanogenic, halophilic and hyperthermophilic archaea, halophilic bacteria, yeasts, plants, insects, and fishes (e.g., Roberts 2000; Roeßler and Müller 2001; Grant 2004; Hincha and Hagemann 2004). This suggests the synthesis of compatible solutes as a potential universal metabolic strategy for hypersaline, desiccation or low temperature conditions. Moreover, the effect of osmolyte synthesis could be doubly advantageous, because of the property of these substances of sustaining latency in some organisms (Welsh 2000).

We therefore propose that a future europan lander should prospect for the signature of compatible solutes and that lenticulae and chaos features are the most promising places for such inspection. The detection of compatible solutes can be performed through Raman spectroscopy, as has been shown for trehalose in Antarctic samples (Wynn-Williams and Edwards 2000; Edwards et al. 2005). Aqueous solutions of compatible solutes have a relatively wide range of eutectic temperatures (for example, trehalose and glycerol have eutectic temperatures of 244 and $227 \mathrm{~K}$ respectively). So, although eutectic temperature can be lowered further by adding other impurities (e,g., salts), chaos regions where close diapirism seems to have occurred (Schenk and Pappalardo 2004) would have more potential for detection of low-temperature eutectic substances than isolated lenticulae, where the effects of thermal plumes must be more limited. Thus, chaos and lenticulae are major objectives for the search of biomarkers on Europa, and maybe for understanding the evolution of the habitability of its upper ice shell.

Acknowledgements We thank Delphine Nna Nvondo for their comments, José I. Belio for drawing Fig. 3, and Pati for her encouragement during the realization of this work. I also thank the suggestions from an anonymous reviewer. Work by JR was supported by a contract I3P with the CSIC co-financed by the Fondo Social Europeo. Work by LM was supported by a grant from the Consejo Nacional de Ciencia y Tecnología of Mexico, CONACYT (Ref-178909).

\section{References}

Bodnar RJ (2001) PTX phase equilibria in the $\mathrm{H}_{2} \mathrm{O}-\mathrm{CO}_{2}$ salt system at Mars near-surface conditions. Lunar and Planetary Science Conference XXXII, abstract 1689

Carlson RW, Johnson RE, Anderson MS (1999) Sulfuric acid on Europa and the radiolytic sulfur cycle. Science 286:97-99

Chyba CF (2000) Energy for microbial life on Europa. Nature 403:381-382

Chyba CF, Phillips CB (2001) Possible Ecosystems and the search for life on Europa. Proc Natl Acad Sci U S A 98:801-804

Chyba CF, Phillips CB (2002) Europa as an above of life. Orig Life Evol Biosph 32:47-68

Cleland D, Krader P, McCree C, Tang J, Emerson D (2004) Glycine betaine as a crioprotectant for prokaryotes. J Microbiol Methods 58:31-38

Dash JG, Haiying F, Wettleufer JS (1995) The premelting of ice and its environment consequences. Rep Prog Phys 58:115-167 
DasSarma S, Arora P (2002) Halophiles. In: Encyclopedia of life sciences, volume 8. Nature, London, pp 458-466

Deming JW (2002) Psychrophiles and polar regions. Curr Opin Microbiol 5:301-309

Edwards HGM, Moody CD, Jorge-Villar SE, Wynn-Williams DD (2005) Raman spectroscopic detection of key biomarkers of cyanobacteria and lichen symbiosis in extreme Antarctic habitats: evaluation for Mars Lander missions. Icarus 174:560-571

Elbein AD, Pan YT, Pasttuszak I, Carroll D (2003) New insights on trehalose: a multifunctional molecule. Glycobiology 13:17-27

Figueredo PH, Greeley R (2004) Resurfacic history of Europa from pole-to-pole geologic mapping. Icarus 167:287-312

Figueredo PH, Greeley R, Neuer S, Irwin L, Schulze-Makuch D (2003) Locating potential biosignatures on Europa from surface geology observations. Astrobiology 3:851-861

Gaidos EJ, Nealson KH, Kirschvink JL (1999) Life in ice-covered oceans. Science 284:1631-1633

Grant WD (2004) Life at low water activity. Philos Trans R Soc Lond B 359:1249-1267

Greeley R, Chyba CF, Head JW, McCord TB, McKinnon WB, Pappalardo RT, Figueredo PH (2004) Geology of Europa. In: Bagenal F, et al. (eds) Jupiter: the planet, satellites and magnetosphere. Cambridge University Press, Cambridge, pp 329-362

Greenberg R, Geissler P, Tufts BR, Hoppa GV (2000) Habitability of Europa's crust: the role of tidal-tectonic processes. J Geophys Res 105:17,551-17,562

Hincha DK, Hagemann M (2004) Stabilization of model membranes during drying by compatible solutes involved in the stress tolerance of plants and microorganisms. Biochem J 383:277-283

Hussmann H, Spohn T (2004) Thermal-orbital evolution of Io and Europa. Icarus 171:391-410

Jaeger JC (1964) Thermal effects of intrusions. Rev Geophys 2:443-466

Jaeger JC (1968) Cooling and solidification of igneous rocks. In: Hess HH, Poldervaart A (eds) Basalts: the Poldervaart treatise on rocks of igneous composition, volume 2. Wiley, New York, pp 503-536

Kadel SD, Chuang FC, Greeley R, Moore JM, The Galileo SSI Team (2000) Geologic history of the Tyre region of Europa: a regional perspective on Europan surface features and ice thickness. J Geophys Res $105: 22,657-22,669$

Kargel JS, Kaye JZ, Head JW, Marion GM, Sassen R, Crowley JK, Ballesteros OP, Grant SA, Hogenboom DL (2000) Europa's crust and ocean: origin, composition, and the prospects for life. Icarus 148:226-265

Kempf B, Bremer E (1998) Uptake and synthesis of compatible solutes as microbial stress responses to highosmolarity environments. Arch Microbiol 170:319-330

Kirk RL, Stevenson DJ (1987) Thermal evolution of a differenciated Ganymede and implications for surface features. Icarus 69:91-134

Kivelson MG, Khurana KK, Russell CT, Volwerk M, Walker RJ, Zimmer C (2000) Galileo magnetometer measurements: a stronger case for a subsurface ocean at Europa. Science 289:1340-1343

Lipps JH, Rieboldt S (2005) Habitats and taphonomy of Europa. Icarus 177:515-527

Marion GM, Fritsen CH, Eicken H, Payne MC (2003) The search for life on Europa: limiting environmental factors, potential habitats, and Earth analogues. Astrobiology 3:785-811

McCord TB, Hansen GB, Fanale FP, Carlson RW, Matson DL, Johnson TV, Smythe WD, Crowley JK, Martin PD, Ocampo AR, Hibbitts CA, Granahan JC (1998) Salts on Europa's surface detected by Galileo's near-infrared mapping spectrometer. Science 280:1242-1245

McKinnon WB (1999) Convective instability in Europa's floating ice shell. Geophys Res Lett 26:951-954

Nimmo F, Manga N (2002) Causes, characteristics and consequences of convective diapirism on Europa. Geophys Res Lett 29:2109 (10.1029/2002GL015754)

Pappalardo RT, Barr AC (2004) The origin of domes on Europa: the role of thermally induced compositional diapirism. Geophys Res Lett 31:L01701 (10.1029/2003GL019202)

Pappalardo RT, Belton MJS, Breneman HH, Carr MH, Chapman CR, Collins GC, Denk T, Fagents S, Geissler PE, Giese B, Greeley R, Greenberg R, Head JW, Helfenstein P, Hoppa G, Kadel SD, Klaasen KP, Klemaszewski JE, Magee K, McEwen AS, Moore JM, Moore WB, Neukum G, Phillips CB, Prockter LM, Shubert G, Senske DA, Sullivan RJ, Tufts BR, Turtle EP, Wagner R, Williams KK (1999) Does Europa have a subsurface ocean? Evaluation of the geological evidence. J Geophys Res 104:24,015-24,055

Pappalardo RT, Head JW (2001) The thick-shell model of Europa's geology: implications for crustal processes. Lunar and Planetary Science Conference XXXII, abstract 1866

Pappalardo RT, Head JW, Greeley R, Sullivan RJ, Pilcher C, Schubert G, Moore WB, Carr MH, Moore JM, Belton MJS, Goldsby DL (1998) Geological evidence for solid-state convection in Europa's ice shell. Nature 391:365-368

Potts M (1994) Desiccation tolerance of prokaryotes. Microbiol Rev 58:755-805

Price PB (2000) A habitat for psychrophiles in deep Antartic ice. Proc Natl Acad Sci U S A 97:1247-1251 
Price PB (2003) Life in solid ice on Earth and other planetary bodies, in Bioastronomy 2002: Life among the stars. In: Norris R and Stootman F (eds) IAU Symposium series no. 213, pp 363-366

Price PB, Sowers T (2004) Temperature dependence of metabolic rates for microbial growth, maintenance, and survival. Proc Natl Acad Sci U S A 101:4631-4636

Prockter LM, Antman AM, Pappalardo RT, Head JW, Collins GC (1999) Europa: stratigraphy and geological history of the anti-Jovian region from Galileo E14 solid-state imaging data. J Geophys Res 104:16,53116,540

Reynolds RT, Squyres SW, Colburn DS, McKay CP (1983) On the habitability of Europa. Icarus 56:246-254

Roberts MF (2000) Osmoadaptation and osmoregulation in Archaea. Front Biosci 5:796-812

Roeßler M, Müller V (2001) Osmoadaptation in bacteria and archaea: common principles and differences. Environ Microbiol 3:743-754

Rothschild LJ, Mancinelli RL (2001) Life in extreme environments. Nature 409:1092-1101

Ruiz J (2005) The heat flow of Europa. Icarus 177:438-446

Ruiz J, Tejero R (2000) Heat flows through the ice lithosphere of Europa. J Geophys Res 105:23,283-23,289

Ruiz J, Tejero R (2003) Heat flow, lenticulae spacing, and possibility of convection in the ice shell of Europa. Icarus 162:362-373

Schenk PM, Pappalardo RT (2004) Topographic variations in chaos on Europa: implications for diapiric formation. Geophys Res Lett 31:L16703 (10.1029/2004GL019978)

Schilling N, Khurana KK, Kivelson MG (2004) Limits on an intrinsic dipole moment in Europa. J Geophys Res 109:E05006 (10.1029/2003JE002166)

Schulze-Makuch D, Irwin LN (2002) Energy cycling and hypothetical organisms in Europa's ocean. Astrobiology 2:105-121

Sotin C, Head JW, Tobie G (2002) Europa: tidal heating of upwelling thermal plumes and the origin of lenticulae and chaos melting. Geophys Res Lett 29 (10.1029/2001GL013844)

Spaun NA, Head JW, Pappalardo RT (2004) Europan chaos and lenticulae: a synthesis of size, spacing, and areal density analyses. Lunar and Planetary Science Conference XXXV, abstract 1409

Spaun NA, Head JW, Collins GC, Prockter LM, Pappalardo RT (1998) Conamara chaos region, Europa: reconstruction of mobile polygonal ice blocks. Geophys Res Lett 25:4277-4280

Spencer JR, Grundy WM, Dumas C, Carlson RW, McCord TB, Hansen GB, Terrile RJ (2006) The nature of Europa's dark non-ice surface material: spatially-resolved high spectral resolution spectroscopy from the Keck telescope. Icarus 182:202-210

Stan-Lotter H, Radax C, Gruber C, Legat A, Pfaffenhuemer M, Wieland H, Leuko S, Weidler G, Kömle N, Kargl G (2003) Astrobiology with haloarchaea from Permo-Triassic rock salt. Int J Astrobiol 4:271-284

Thomas DN, Dieckmann GS (2002) Antarctic sea ice - a habitat for extremophiles. Science 295:641-644

Tobie G, Choblet G, Sotin C (2003) Tidally heated convection: constraints on Europa's ice shell thickness. J Geophys Res 108:5124 (10.1029/2003JE002099)

Welsh DT (2000) Ecological significance of compatible solute accumulation by micro-organisms: from single cells to global climate. FEMS Microbiol Rev 24:263-290

Wynn-Williams DD, Edwards HGM (2000) Proximal analysis of regolith habitats and protective biomolecules in situ by laser Raman spectroscopy: overview of terrestrial Antarctic habitats and Mars analogs. Icarus 144:486-503

Yancey PH (2005) Organic osmolytes as compatible, metabolic and counteracting cytoprotectants in high osmolarity and other stresses. J Exp Biol 208:2819-2830 